TTR

Traduction, terminologie, re?daction

\title{
La littérature canadienne-anglaise pour la jeunnesse en traduction québécoise : analyse discursive de la politique éditoriale et de la réception critique des « Deux Solitudes/Jeunesse » (1980-1992)
}

\section{Claire le Brun}

Volume 7, numéro 1, 1er semestre 1994

Genres littéraires et traduction

URI : https://id.erudit.org/iderudit/037172ar

DOI : https://doi.org/10.7202/037172ar

Aller au sommaire du numéro

Éditeur(s)

Association canadienne de traductologie

ISSN

0835-8443 (imprimé)

1708-2188 (numérique)

Découvrir la revue

Citer cet article

le Brun, C. (1994). La littérature canadienne-anglaise pour la jeunnesse en traduction québécoise : analyse discursive de la politique éditoriale et de la réception critique des « Deux Solitudes/Jeunesse » (1980-1992). TTR, 7(1), 153-189. https://doi.org/10.7202/037172ar
Résumé de l'article

La Littérature canadienne-anglaise pour la jeunesse en traduction québécoise: analyse discursive de la politique éditoriale et de la réception critique des " Deux Solitudes/Jeunesse » (1980-1992). La collection « Les Deux

Solitudes/Jeunesse ", publiée aux éditions Pierre Tisseyre à Montréal, est un cas exemplaire de traduction de littérature canadienne-anglaise pour la jeunesse en français, puisque c'est la seule qui se donne pour objectif de faire connaître au Québec les « oeuvres marquantes » du Canada anglais. L'étude fait l'historique de la collection (1980-1992) et analyse le discours justificatif et les choix éditoriaux. Le contenu discursif des textes traduits est comparé à celui des textes publiés en français au Québec durant la même période. La dernière partie examine la réception critique de la collection dans les revues spécialisées: la reconnaissance ou non du statut de traduction du texte, les paramètres d'évaluation utilisés par les critiques. La conclusion met l'accent sur une évolution, dans les choix de traduction comme dans la réception critique, du discours de la différence vers un discours de la communauté d'expérience des jeunes. Cette tendance assimilatrice est particulièrement perceptible dans la traduction des renvois intertextuels et des références culturelles, dont l'analyse est esquissée et qui sera poursuivie dans un autre cadre.

Tous droits réservés ( $\mathrm{C}$ TTR: traduction, terminologie, rédaction — Les auteurs, Ce document est protégé par la loi sur le droit d’auteur. L’utilisation des 1994 services d'Érudit (y compris la reproduction) est assujettie à sa politique d'utilisation que vous pouvez consulter en ligne.

https://apropos.erudit.org/fr/usagers/politique-dutilisation/ 


\section{La littérature canadienne-anglaise pour la jeunesse en traduction québécoise: analyse discursive de la politique éditoriale et de la réception critique des «Deux Solitudes/Jeunesse» (1980-1992)}

\section{Claire le Brun}

Dans le domaine de la littérature canadienne pour la jeunesse, la traduction d'une langue officielle à l'autre a longtemps occupé une place restreinte. L'utile enquête d'André Gagnon (1987), couvrant les années 1900-1986, fait ressortir des chiffres éloquents: 14 livres traduits de 1900 à 1959 et 19 de 1960 à 1971. En opposition, la dernière période 1972-1986 est celle d'une extraordinaire explosion, puisque 323 livres ont été traduits au cours de ces quinze années. L'auteur de l'étude note cependant une forte disproportion entre les traductions vers l'anglais et celles vers le français: 65 dans le premier cas et 258 dans le second, soit quatre fois plus ${ }^{1}$. Fondéc en 1980 à Montréal, la collection Les Deux Solitudes/Jeunesse participe de ce vaste

1. L'histoire des mouvements de traduction de la littérature pour la jeunesse ne reflète donc pas celle de la traduction des livres canadiens dans leur ensemble. Selon Philip Stratford (1977, p.

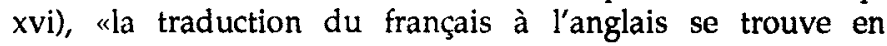
meilleure posture que sa contrepartie de l'anglais au français». Pour un panorama de la traduction littéraire au Canada, voir Mezei (1988), Martin (1992, ainsi que sa communication du colloque de l'ACT en 1993). 
mouvement de traduction de la littérature canadienne-anglaise pour la jeunesse en français. Elle est cependant la seule à avoir comme programme éditorial la diffusion au Québec d'une sélection de textes pour la jeunesse provenant du Canada anglais.

L'analyse de cette collection exemplaire, de 1980 à 1992, qui fait partie d'une étude générale sur la traduction de textes de langue anglaise dans l'édition francophone pour la jeunesse ${ }^{2}$, s'ordonne autour de quatre axes: 1. données de sociologie externe: le milieu, les agents; 2 . évolution de la politique éditoriale; 3. la littérature pour la jeunesse et ses discours; 4. réception critique de la traduction. En conclusion, nous nous interrogerons sur l'évolution des principes de traduction au cours de la période étudiée.

\section{Données de sociologie externe}

\subsection{Naissance de la collection}

La collection Les Deux Solitudes/Jeunesse est la petite sœur de la collection Les Deux Solitudes, fondée par Michelle Tisseyre en 1974 au Cercle du Livre de France (éditeur Pierre Tisseyre), et qui a traduit les Morley Callaghan, Robertson Davies, Margaret Laurence, Mordecaï Richler. Le soutien institutionnel dont jouissent ces collections doit être souligné. Rappelons le texte de

2. Cette recherche est le prolongement naturel d'une étude antérieure de la littérature québécoise pour la jeunesse, dans la perspective de l'analyse discursive. Travaillant sur l'ensemble des romans pour la jeunesse parus au Québec entre 1970 et 1990, nous avions inclus dans notre corpus les traductions de l'anglais, qui en faisaient partie en termes de réception. Il importait de voir comment le discours constitué par cette juxtaposition de textes traduits s'articulait avec le discours des textes québécois produits à la même époque. Les observations qui en ont résulté ont montré l'intérêt d'une recherche distincte sur le phénomène de la traduction en littérature pour la jeunesse. 
présentation, reproduit dans tous les ouvrages des deux collections:

Grâce à un programme d'aide à la traduction du Conseil des Arts, il est enfin devenu possible de faire connaître au Québec les œuvres marquantes d'auteurs canadiens-anglais connues souvent dans tous les pays de langue anglaise, mais ignorées dans les pays de langue française parce qu'elles n'avaient jamais été traduites.

$\mathrm{Ce}$ même programme va permettre aux œuvres marquantes de nos écrivains d'être traduites en anglais.

La Collection des Deux Solitudes a donc pour but de faire connaître, en français, les ouvrages les plus importants de la littérature canadienne de ces dernières années.

La collection Les Deux Solitudes Jeunesse est lancée officiellement le 6 mai 1980 à Montréal, en pleine période référendaire. Les éditions Pierre Tisseyre avaient effectué quelques coups de sonde, les années précédentes, en publiant, de 1976 à 1979, des traductions d'albums ou de romans de Robert Allen, Mordecaï Richler, Barbara Smucker, Margaret Atwood. A ceux-ci s'ajouteront, pour le lancement, quatre titres: un Morley Callaghan, un Jean Little et deux Farley Mowat.

\subsection{Le discours justificatif}

Le texte de présentation, cité plus haut, met de l'avant deux arguments: le manque à combler et la promesse $d^{\prime} u n$ échange. Le manque est l'ignorance, à cause d'un obstacle linguistique, de textes canadiens-anglais dont la valeur est attestée par leur pénétration dans tous les pays de langue anglaise. La promesse est celle d'une réciproque, puisque nos écrivains seront à leur tour traduits en anglais.

Dans un article paru dans Canadian Children's Literature/Littérature canadienne pour la jeunesse (CCL), revue de 
critiques et d'études de la littérature canadienne pour la jeunesse ${ }^{3}$, Paule Daveluy (1980 a, p. 6), directrice de la nouvelle collection, ajoute deux autres arguments: son utilité pédagogique dans un objectif de bilinguisme et son importance stratégique sur la scène éditoriale internationale. La collection profitera non seulement aux jeunes francophones du Québec, mais à tous les francophones du Canada et aux anglophones des classes d'immersion, qui «apprendront le français en lisant non seulement Ginette Anfousse et Claude Aubry mais également, bénéfice inattendu, Farley Mowat et Barbara Smucker». Selon Paule Daveluy, ces textes en traduction s'ajouteront aux livres québécois pour «faciliter le bilinguisme aux jeunes Canadiens». Par ailleurs, il y a une place à reprendre dans le marché du livre. Le Canada a laissé l'Étranger - Paris, Londres, New York - le supplanter dans l'édition des traductions de son patrimoine: «il est temps que nos traducteurs et éditeurs locaux remplacent les traducteurs et éditeurs étrangers dans ce travail de repossession de nos richesses».

Notons enfin que la jeune collection est portée par un vent de bilinguisme en littérature pour la jeunesse. $A$ la fin de 1980, dans le numéro même où paraît l'article de Paule Daveluy, la revue $C C L$ annonce son nouveau contenu bilingue. La présentation des Deux Solitudes/Jeunesse y occupe une place de choix, en vis-à-vis de l' éditorial qui établit un lien explicite entre le récent référendum et la nouvelle orientation de la revue.

A partir de 1990, le texte de présentation, légèrement modifié, fait le bilan de la collection: «Déjà plus d'une trentaine d'ouvrages, choisis pour leur qualité, leur intérêt et leur originalité font honneur à cette collection [...]». Il est également précisé que la collection s'adresse aux jeunes francophones du Québec et des autres provinces.

3. Les idées de Paule Daveluy sur la traduction de la littérature de jeunesse au Canada sont également exposées dans Daveluy (1980b) et dans Robin (1981). 


\subsection{Les agents}

\subsubsection{L'éditeur et la directrice de collection}

Le Cercle du Livre de France et l'éditeur Pierre Tisseyre ont sur la scène montréalaise une image d'ouverture aux littératures hors-Québec. L'intérêt pour la littérature canadienne s'affirme dès 1974 avec la création de la collection Les Deux Solitudes, référence au célèbre Two Solitudes de Hugh Mac Lennan (1945). Le programme de subventions aux traductions du Conseil des Arts, rappelons-le, a été créé en 1972 par Gérard Pelletier ${ }^{4}$. À la fin des années 70, le Cercle du Livre de France devient: Pierre Tisseyre éditeur ${ }^{5}$.

Paule Daveluy, la directrice-fondatrice de la collection, a derrière elle une longue carrière d'auteure pour la jeunesse. Son premier roman Un été enchanté, paru à Montréal en 1959, avait connu une excellente réception critique. Il a été traduit et publié aux États-Unis en 1962, puis publié en Grande-Bretagne. Daveluy compte parmi les premières promotrices de la littérature québécoise pour la jeunesse; elle est membre-fondateur et première présidente de l'organisme Communication Jeunesse en $1971^{6}$. Elle a remporté plusieurs prix. Le début de sa carrière de traductrice littéraire coïncide à peu près avec la direction de la collection. La traduction est pour l'auteure une nouvelle forme d'écriture pour la jeunesse? ${ }^{7}$. Marie-Andrée Clermont, qui lui

4. Sur le contexte institutionnel de la traduction au Canada, voir Martin (1992).

5. Sur les rapports des deux maisons d'édition, voir Huard (1982).

6. Sur l'historique de la littérature pour la jeunesse de langue française au Canada et particulièrement sur l'action de Paule Daveluy, voir Lemieux (1972), pp. 151-153 et 283-285.

7. Voir l'entrevue accordée à Marie-Jeanne Robin pour Lurelu (Robin 1981, p. 16): "Il m'est difficile de créer [...]. Je préfère jouer avec les mots, avec les phrases. Et je trouve ce plaisir 
succédera en 1990, a publié son premier roman pour la jeunesse en 1980 et sa première traduction d'un roman jeunesse (Monica Hughes) en 1984. Elle a fait sa première apparition comme traductrice de la collection en $1986^{8}$.

\subsubsection{Les traducteurs}

On peut discerner deux profils de traducteurs: les traducteurs littéraires; les écrivains et traducteurs pour la jeunesse (dans cet ordre). Le second profil, qui est celui des deux directrices de collection, est le plus important quantitativement et a une présence constante pendant toute la durée de la collection.

Les traducteurs littéraires sont en général ceux de la collection Les Deux Solitudes: Jean Simard, Claire Martin, Michelle Tisseyre, Michelle Robinson. Ils se spécialisent dans la traduction du roman canadien. Les traducteurs pour la jeunesse tirent généralement leur légitimité de leur succès comme écrivains pour la jeunesse: les Claude Aubry, Michel Caillol, Maryse Côté, Marie-Andrée Clermont, Cécile Gagnon ont vu leur talent consacré par des prix. Le cas de Claude Aubry, décédé en 1985, est exemplaire à cet égard. Auteur primé, traduit au Canada anglais, «parfait bilingue», il a donné son nom à un prix prestigicux "plus connu au Canada anglais qu'au Canada français», selon l'in memoriam rédigé par Paule Daveluy pour la revue $C C L$ (Daveluy 1985). Il représente cet idéal d'auteur canadien voué à la rencontre des "deux solitudes». Il faut noter cependant que le début des années 90 semble annoncer une

dans mon travail de traduction et de révision.»

8. Interrogée sur les places respectives de la traduction et de l'écriture dans son activité, l'auteure répond: «La traduction me permet de gagner ma vie, l'écriture de m'offrir un plaisir. Grâce à la traduction, j'ai acquis une approche scientifique de la recherche [...]. La traduction s'avère donc une bonne base pour l'écriture [...]. En fait, je suis quelqu'un qui travaille pour les jeunes. Les modalités en sont l'écriture et la traduction.» (Poulin 1987, p. 16) 
nouvelle génération de traductrices, lectrices plus qu'auteures de littérature pour la jeunesse, telle Martine Gagnon.

Quelques chiffres donneront une idée de la présence relative des traducteurs dans la collection. Douze traducteurs sont intervenus depuis la fondation. Les deux directrices de collection sont aussi les traductrices les plus actives: Paule Daveluy a traduit 14 romans de 1978 à 1992. Marie-Andrée Clermont, 7 romans entre 1986 et 1992. Puis vient Michelle Tisseyre, directrice générale de la collection «Les Deux Solitudes», qui a signé quatre traductions entre 1980 et 1989 . Les neuf autres traducteurs ont effectué une traduction: Claire Martin, Jean Simard, Hélène Filion, Cécile Gagnon; deux traductions: Michel Caillol, Michelle Robinson, Martine Gagnon; trois traductions: Maryse Côté et Claude Aubry (voir annexe).

\section{2. Évolution de la politique éditoriale}

La question des critères de choix des textes est d'une importance majeure pour l'analyse discursive. Du choix même de la directrice de collection d'ordonner dans la même catégorie éditoriale tel roman de Lucy Maud Montgomery, de Farley Mowat, de Claire Mackay, de Kevin Major va naître un système - parmi les systèmes possibles - de représentations du Canada anglais à l'intention des jeunes lecteurs francophones. Paule Daveluy (1980) a brièvement abordé la question du choix des textes à traduire dans son article de présentation. Confrontons ces principes de départ à l'évolution de la collection de 1980 à 1992.

\subsection{Principes éditoriaux}

Paule Daveluy mentionne deux critères: le premier est, comme on pourrait $s^{\prime} y$ attendre, l'obtention d'un prix littéraire dans la culture-source. Le second, qui concerne l'ensemble de la collection, est l'équilibre, en nombre de titres, entre les auteurs consacrés de la littérature générale qui écrivent aussi pour la jeunesse et ceux qui se spécialisent dans la littérature pour la jeunesse. 
La directrice fait état $d$ 'un «corpus considérable» de livres candidats à la traduction et établit une comparaison implicite entre le Canada anglais et le Canada français, au désavantage de ce dernier: «Au Canada anglais, les auteurs dits "pour adultes» ne dédaignent pas d'écrire pour les jeunes» (Daveluy $1982 \mathrm{a}, \mathrm{p}$. 7).

\subsection{Les titres de lancement: 1976-1980}

Les quatre titres précédant le lancement officiel de la collection se répartissent comme suit: deux albums, le Violon (1976) de Robert Allen, paru presque simultanément en anglais et en français, et Sur un arbre perchés (1978) de Margaret Atwood; un court roman humoristique de Mordecaï Richler, Jacob Deux-Deux (1977) et un roman à portée sociale de Barbara Smucker, les Chemins secrets de la liberté (1979). Dans tous les cas, il s'agit de livres récents; l'écart maximum entre la parution en anglais et la traduction est de deux ans (voir annexe). Les textes traduits tirent leur légitimité de la notoriété des auteurs auprès des francophones (Atwood, Richler) ${ }^{9}$ ou de l'importance du sujet traité: le roman de Smucker évoque la fuite d'esclaves noirs américains au Canada au XIXe siècle.

Sur les quatre titres du lancement officiel en mai 1980, trois sont des classiques: les textes originaux de la Promesse de Luke Baldwin (Luke Baldwin's Vow) de Morley Callaghan, de Deux grands ducs dans la famille (Owls in the Family) et de la Malédiction du tombeau viking (The Curse of the Viking Grave) de Farley Mowat sont parus respectivement en 1948, 1961 et 1966. On pourrait parler pour ceux-là de rattrapage ${ }^{10}$. Le quatrième est récent:

9. Selon Ruth V. Martin (1982, pp. 19-20), M. Atwood, M. Callaghan et $M$. Richler sont parmi les cinq auteurs les plus traduits pendant la période 1967-1982; les deux autres auteurs étant R. Davies et M. Laurence.

10. Une traduction française de Lost in the Barrens (1956), Perdus dans le Grand Nord, est parue en 1974 aux éditions Robert Laffont (Paris). Il ne restait plus aux traducteurs canadiens que 
Écoute, l'oiseau chantera (Listen for the Singing) de Jean Little a remporté le prix du Canada Council Children's Literature (CCCL) en 1977. Encore faut-il préciser que l'action de ce roman se situe durant la seconde Guerre mondiale. Aux yeux des lecteurs, il rejoindra vraisemblablement les trois autres dans un effet de décalage temporel.

\subsection{1-1989: la direction de Paule Daveluy}

Sur la vingtaine de titres parus au cours de ces années, la plupart ont remporté des prix, notamment celui du CCCL ou celui du CLA. Deux tendances éditoriales coexistent: la traduction de classiques - Une goélette nommée Black Joke (The Black Joke) de Farley Mowat (1962) et le cycle d'Emily de Lucy Maud Montgomery (1925-27) - et la traduction de prix et de succès critiques récents. L'écart entre la publication en langue originale et la traduction varie entre deux et sept ans, avec une moyenne de trois ans (voir annexe). Plusieurs auteurs reviennent deux ou trois fois: Brian Doyle, Bill Freeman, Monica Hughes, Jean Little, Claire Mackay, Barbara Smucker. Parmi les romans de tel ou tel auteur, certains choix ou certaines absences peuvent étonner. Ainsi, dans le cas de Brian Doyle, trois romans ont été retenus, dont Up to Low (1982), qui a remporté un prix (CLA) mais qui a été diversement accueilli par la critique, mais pas Angel Square (1985), qui traite avec pertinence des affrontements de rues entre les jeunes «Pea soups", «Dogans» ou "Jews" dans l'Ottawa de la seconde Guerre mondiale et paraît ainsi convenir aux objectifs de la collection ${ }^{11}$.

la suite, The Curse of the Viking Grave, généralement jugée inférieure. Le commentaire suivant d'une bibliothécaire de Hull (Lepage 1980) exprime une opinion répandue: "le Canada anglais a de bien meilleurs romans à offrir aux jeunes francophones».

11. "In spite of the scenes of child violence, however, which are not to be taken literally, the overall tone is one of joy, reconciliation, generosity, and acceptance. Angel Square is a very warm-hearted story and Tommy is the most lovable young hero 


\section{4. À partir de 1990: la direction de Marie-Andrée Clermont}

À la fin de 1989, la collection passe au format poche et numérote ses titres. Dès le numéro 4, la direction passe à Marie-Andrée Clermont, traductrice de la collection depuis 1986. Trois tendances caractérisent pour le moment la nouvelle direction:

1. Réédition de titres: Émilie de L. M. Montgomery (4 volumes), la Fille à la mini-moto de Claire Mackay et Je t'attends à Peggy's Cove de Brian Doyle. Ces traductions, de Paule Daveluy et de Claude Aubry respectivement, ont obtenu des prix. Claude Aubry a obtenu le prix du Conseil des arts, une première en littérature pour la jeunesse!

2. Traductions d'auteurs déjà présents dans la collection: les numéros 1 et 2 sont des Kevin Major, apparu pour la première fois dans la collection en 1984 avec Tiens bon!. Brian Doyle réapparaît avec la Vie facile.

3. Découverte d'auteurs, récemment apparus dans la culture-source: Marilyn Halvorson, William Bell, Audrey O'Hearn sont représentatifs de cette nouvelle génération.

2.5. Statut des auteurs et des textes: décalage entre la culturesource et la culture-cible? Le melting-pot éditorial

Comme cette collection est l'unique structure éditoriale québécoise consacrée à la littérature pour la jeunesse canadienne anglaise, elle fait voisiner des textes appartenant à des époques, des catégories éditoriales différentes et destinées à différentes classes d'âge. Elle met aussi sur le même plan des auteurs jouissant de statuts différents dans la culture-source.

\subsection{1. Époque des versions originales}

Les dates de parution des textes anglais varient de 1925 (Emily) à 1990 (sondage effectué jusqu'au n 20, 1992).

in Canadian children's literature., (Egoff et Saltman 1990, p. 46) 


\subsubsection{Catégories éditoriales}

La plupart des récits ont une portée éducative, mais il se glisse aussi quelques titres classés divertissement («escapist literature», "popular literature» dans la culture-source): la Fille à la mini-moto étiqueté «sweet dream romance» ou Terreur au bivouac (E. Wilson), étiqueté «mystery story ${ }^{12} »$. La réédition du premier titre semble indiquer un grand succès de librairie.

\subsubsection{Classes d'âge}

C'est à ce niveau qu'apparaît la plus grande disparité. À ses débuts la collection a édité deux albums pour enfants. Elle a ensuite publié simultanément des récits pour enfants et pour adolescents. La nouvelle formule inclut le roman pour jeunes adultes. À partir de 1988, des mentions d'âge sont indiquées, de façon irrégulière et fort discrètement, avec les données de catalogage: «pour les 10-14 ans», "pour les 12-14 ans», "pour enfants", "pour adolescents", "pour jeunes». Les Deux Solitudes/Jeunesse s'écartent ainsi de la tendance actuelle en littérature de jeunesse québécoise qui délimite de plus en plus précisément le lectorat des collections.

L'évolution de la collection, de 1980 à 1992 , pourrait donc se résumer ainsi: après un hommage rétrospectif, voire posthume, à quelques grands auteurs de la littérature canadienne-anglaise, les traductions suivent de près la production actuelle.

\section{Les discours en présence: romans québécois et romans canadiens-anglais traduits}

L'examen de la politique éditoriale ayant fait apparaître ces deux tendances successives, on peut en déduire qu'au fil des années, le discours des traductions et celui des romans québécois tendent à se rejoindre. Aussi nous paraît-il justifié de maintenir dans cette partie la commode périodisation par décennie.

12. Nous nous référons ici à Egoff et Saltman (1990) et aux critiques parues dans CCL et In Review. 


\subsection{Les années 80}

Deux objets du discours prédominent: le passé/l'Histoire et la famille. Les récits appartiennent aux catégories, bien représentées dans la culture-source, Historical fiction et Stories of child and family life (Egoff et Saltman, 1990).

Le récit historique occupe une place très importante dans la littérature canadienne-anglaise pour la jeunesse ${ }^{13}$. Certains $y$ voient la marque du sérieux de cette littérature (Denton 1983)! Le récit historique représenté dans le corpus de traductions présente deux caractéristiques, dont l'importance relative varie selon les récits: l'aventure et la peinture sociale. Les épopées du Grand Nord et de la marine - surtout à voile - inspirent six des vingt titres des années 1981-89 (Mowat, Clark, Freeman, German, Lunn). Parmi les romans où domine une préoccupation sociale se classent la Passion de Blaine de Monica Hughes, roman d'apprentissage ayant pour cadre l'Ontario de la Grande Dépression et de l'avant-guerre, et surtout les Chemins de la liberté et Jours de terreur de Barbara Smucker, qui dénoncent l'oppression, respectivement celle des Noirs américains et celle des Mennonites chassés par la Révolution russe. Dans ces deux récits, le Canada devient la terre d'accueil des opprimés. Il faut noter que toutes les aventures historiques du corpus comportent des éléments de réflexion sur la société. Bill Freeman et Farley Mowat (Une goélette nommée Black Joke) peignent la misère des populations de marins et de pêcheurs, l'un à la fin du XIX' siècle, l'autre dans les années 30. Enfin, le métissage entre les Blancs et les Amérindiens est un motif récurrent, qui prend un relief particulier chez Mowat (la Malédiction du tombeau viking) et chez Lunn (Une ombre dans la baie).

L'analyse comparative du discours sur l'Histoire dans la littérature canadienne pour la jeunesse du côté anglais et du côté français est une vaste question que nous ne pouvons qu'effleurer dans les limites du présent article. C'est sur cet objet que l'on

13. Voir Egoff et Saltman (1990, pp. 103-130), Atkinson (1979, 1981) et plusicurs articles et recensions parus dans $C C L$. 
peut s'attendre à trouver le plus de divergences entre les deux discours. Le récit historique a été illustré au Québec dans les années 60 et 70 par les Monique Corriveau, Suzanne Martel, Yves Thériault, Robert de Roquebrune ${ }^{14}$. Le discours portait alors principalement sur le Régime français et sur la rencontre entre les Blancs et les Amérindiens. Quant aux auteurs des années 80 , ils se consacrent massivement à la peinture d'un Québec urbain actuel. Selon nous, la réflexion sur l'Histoire et le politique a été prise en charge au cours de la dernière décennie par des auteurs de science-fiction. Denis Côté, particulièrement, mais également Daniel Sernine, Johanne Massé, Jacques Lazure ${ }^{15}$. La vision de ces auteurs dépasse généralement les frontières nationales. Au Canada anglais, par contre, le récit historique est très vivant dans les années 80, diversifiant ses sujets d'étude. Ainsi un groupe de récits portent sur les mouvements syndicaux. Certains d'entre eux ont pour cadre le Québec: One Proud Summer (M. Hewitt et C. Mackay, 1981) se fonde sur la grève de Valleyfield, en 1939. Trouble at Lachine Mill (Bill Freeman, 1983) décrit les conditions de travail dans l'industrie du textile vers 1870 . Les points de congruence et de discordance entre les deux discours français, le traduit et l'original, sont aisément repérables. Le point d'accord le plus évident est l'intérêt pour l'interaction entre les Blancs et les Amérindiens, le métissage étant toutefois plus explicite chez les auteurs canadiens-anglais. La différence réside dans les périodes et les lieux privilégiés. Nous nous permettrons ici une parenthèse sur les héros-types de la collection, car la remarque

14. M. Corriveau, le Wapiti (1964); S. Martel, Jeanne, fille du Roy (1974); Robert de Roquebrune, la Seigneuresse (1960); les Habits rouges (1978); Yves Thériault, Ashini (1961); le Ru d'Ikoué (1977).

15. D. Côté, Hockeyeurs cybernétiques (1983); l'Invisible puissance (1983); l'Idole des Inactifs (1989); la Révolte des Inactifs (1990); le Retour des Inactifs (1990); le Voyage dans le temps (1989). D. Sernine, Opération Argus (1979); Argus intervient (1983); Argus mission mille (1988); la Nef dans les nuages (1989). J. Massé, De l'autre côté de l'avenir (1985); Contre le temps (1987); le Passé en péril (1990). J. Lazure, le Domaine des Sans-Yeux (1989). 
vaut particulièrement pour les récits historiques: Les Deux Solitudes/Jeunesse donnent à voir un nombre impressionnant de jeunes gens roux, d'origine écossaise! Ils sont au premier plan des récits'de Freeman, Mowat, Clark, German, Lunn et même Smucker, qui oppose dans $U n$ monde hors $d u$ temps les visions du monde d'un jeune MacDonald ${ }^{16}$ et d'un jeune Amish. Curieuse conséquence de l'entreprise de traduction que cet effet de saga, produit par la juxtaposition de ces auteurs et de ces titres au sein $\mathrm{d}^{\prime}$ une même collection, effet qui ne fut sans doute ni recherché ni envisagé par les directrices de la collection! Le portrait stéréotypique du Canadien-anglais se trouve renforcé par la structure éditoriale.

Le second objet discursif en importance est le développement de l'adolescente ou de l'adolescent au sein de la famille. Parmi les épreuves qu'elle ou il doit affronter figure au premicr plan l'abandon des parents, surtout celui du père, par la mort ou le divorce: Je t'attends à Peggy's Cove (B. Doyle), Tiens bon!, Maman va t'acheter un moqueur (J. Little). L'ennemi peut aussi être la maladie: Mike, chasseur de ténèbres (M. Hughes); le milieu social: Jasmine (J. Truss); ou la faible estime de soi: le Projet Minerve (C. Mackay). Dans cette catégorie des Stories of family life, on ne peut manquer de noter une tendance marquée chez les auteurs à situer les héros à l'époque de leur propre enfance, combinant ainsi le roman d'apprentissage et le roman historique. En montant à Low (B. Doyle) se passe dans les années 50 . Écoute, l'oiseau chantera (J. Little) se déroule à Toronto, pendant la guerre de 39-45, dans une famille nouvellement immigrée d'Allemagne et élève ainsi le drame familial au niveau de la tragédie historique.

Enfin quelques récits entrent partiellement dans les deux catégories du récit historique et du roman familial, tout en présentant une troisième caractéristique plus importante. Un monde hors du temps de B. Smucker, sur les communautés Amish, aborde le multiculturalisme de la société canadienne. Quelques

16. Sur la famille du héros: «De vraies têtes de mules. Des roux, c'est tout dire.» (p. 11) 
récits, comme la Fille à la mini-moto (C. Mackay) et Terreur au bivouac (E. Wilson) ont surtout leur intérêt dans le suspense et le mystèrc.

C'est dans la catégorie des récits sur les problèmes d'enfance et d'adolescence que se trouvent le plus de points de congruence entre les textes traduits et les textes originaux. Nous reviendrons sur cet aspect à propos des traductions des années 90 où ce type de récit devient dominant.

\subsection{Le début des années 90 (90-92)}

Sur les quatorze nouveaux titres parus en deux ans - les six autres sont des rééditions ${ }^{17}$ - la grande majorité abordent les problèmes des adolescents et des jeunes adultes dans la société actuelle.

À quelques exceptions près, on note une cohésion remarquable entre ces «romans d'apprentissage» des Deux Solitudes/Jeunesse, format poche. Aux problèmes, oserons-nous dire courants, de la classe moyenne - divorce, drogue, échec scolaire, sexualité - s'ajoutent, par les romans de l'auteur terreneuvien Kevin Major, ceux des classes défavorisées. Suivant une tendance amorcée au Canada, français et anglais, dans les années 80 , les récits s'adressent de plus en plus souvent aux jeunes adultes. Emblématique de cette nouvelle collection, Moi et Luc (A. O'Hearn) est un roman de la paternité vécue par le héros, et non plus par le père de celui-ci. Le narrateur de dix-sept ans décide de s'occuper du bébé Luc, que la mère adolescente voulait donner en adoption.

À côté de cette série de récits fortement inscrits dans l'actualité sociale nord-américaine, figurent quelques romans à visée plus ludique, destinés aux lecteurs plus jeunes (le Fantôme de Val-Robert de Beverley Spencer et Les Commandos de la télé de Sonia Kraddock) et deux récits historiques. Ces derniers, Le Ciel

17. C. Mackay, La Fille d̀ la mini-moto; L.M. Montgomery, Émilie de la Nouvelle Lune (4 tomes); B. Doyle, Je t'attends dे Peggy's Cove. 
croule (Kit Pearson) et Shan Da et la cité interdite (William Bell), introduisent des sujets inédits dans le corpus de lecture pour la jeunesse en français. Le roman de Kit Pearson rappelle l'envoi de milliers d'enfants britanniques au Canada pendant la guerre de 39-45. Celui de William Bell évoque les événements récents de la place Tian an Men.

Le discours de cette nouvelle série se rapproche sensiblement du discours des romans québécois publiés au cours des mêmes années: en effet, quelle que soit leur langue d'origine, les textes se rejoignent dans l'universalité de l'expérience de l'adolescence dans la société nord-américaine voire occidentale, au-delà des différences culturelles. On peut se demander si cette apparente diminution de l'altérité des objets du discours exerce une influence sur l'attitude des traductrices et des traducteurs. Nous y reviendrons plus loin.

\section{Réception critique des traductions}

\subsection{Problèmes spécifiques à la collection}

En abordant ce type de corpus, notre question est double. De façon générale, la traduction destinée aux enfants et aux adolescents pose-t-elle des problèmes spécifiques? Plus particulièrement, les objectifs que s'est fixés la collection entraînent-ils des difficultés supplémentaires? A la recherche d'éléments de réponse, nous avons confronté notre propre lecture des traductions à leur réception critique dans les revues spécialisées et au discours des directrices de collection dont il a été question plus haut. Les difficultés apparaissent de plusieurs ordres.

L'écueil le plus souvent signalé dans les recensions critiques est celui des registres. Pour une partie de la question, la problématique est celle de la traduction littéraire en général. La traduction du dialecte de Terre-Neuve dans les dialogues ou la narration à la première personne de Kevin Major relève de la question de la traduction des sociolectes. À cet égard, la collection fournit un terrain d'observation tout à fait riche. En 
effet, les trois romans parus dans la collection ont été traduits par trois traductrices: Michelle Robinson, Marie-Andrée Clermont et Michelle Tisseyre. Si l'on en juge à un ensemble d'indices repérables à la première lecture, la conception de la traduction diffère chez les traductrices. L'étude comparative approfondie de ces trois traductions serait d'un grand intérêt.

Cela dit, dans cette collection à la vocation didactique avouée, la question des registres se pose aussi en termes de lisibilité pour un public que l'on a peut-être voulu trop large.

Comme nous l'avons mentionné plus haut, la collection s'adresse non seulement aux jeunes francophones du Québec et du Canada, mais encore aux jeunes anglophones des classes d'immersion. Dans les recensions, les traductions sont parfois mesurées à l'aune de leur lisibilité par des étudiants en classes d'immersion; le registre de langue peut alors être considéré comme trop élevé. Ne cherche-t-on pas là la quadrature du cercle?

Plus souvent, la question des registres se pose dans le corpus comme disparité entre le registre de la langue-source, jugé simple par les critiques, et celui de la langue d'arrivée, moins accessible, aux yeux de ces derniers. Se greffe alors le problème, que connaissent bien les enseignants de langue seconde, de l'écart entre le niveau de difficulté linguistique et le niveau de difficulté cognitive. Le cas le plus courant est celui du récit que l'âge des héros et la nature de l'intrigue destinent à des enfants, mais qui comporte trop de difficultés linguistiques pour ce public. En bref, la collection Les Deux Solitudes/Jeunesse est, comme toute la littérature pour la jeunesse traduite, soumise a des exigences de lisibilité par la classe d'âge visée par l'auteur du texte-source. Mais, à notre avis, le contrat de lecture s'est dangereusement compliqué avec l'annexation des étudiants en français langue seconde au lectorat de la collection. 


\subsection{Discours critique sur la collection}

\subsubsection{Les revues spécialisées}

Les sondages ont été effectués à partir de trois sources: $C C L$, In Review/Canadian Books for Young People et Lurelu ${ }^{18}$. Les deux premiers périodiques ont une visée pancanadienne et sont bilingues; les critiques de In Review sont bibliothécaires, ceux de $C C L$ ont des appartenances institutionnelles diverses: universitaires (le plus souvent), bibliothécaires et (plus rarement) écrivains pour la jeunesse. Lurelu, uniquement en français, est «la seule rcvue exclusivement consacrée à la littérature québécoise pour la jeunesse», comme l'indique son sous-titre ${ }^{19}$. Il va sans dire que cela a une influence directe sur la conception de la traduction. Les critiques de Lurelu, comme ceux de In Review, sont en grande majorité des bibliothécaires ${ }^{20}$. Précisons encore que, dans Lurelu, les romans de la collection ont été présentés de 1984 à 1986 sous la rubrique «Traductions». Au cours des autres années visées par notre enquête (1978-1983 et 1987-1992) ils ont été traités avec les autres romans, sans distinction. Ce changement éditorial ne semble avoir eu aucune incidence sur le discours critique. Enfin, la revue s'est intéressée à plusieurs reprises à la problématique de la traduction (Paquette 1982; Charette 1985).

18. La revue Québec français, organe de l'Association québécoise des professeurs de français (AQPF), a également été considérée. Nous ne l'avons pas retenue parce que la recension des romans jeunesse y est assurée jusqu'en 1991 par des élèves du primaire et du secondaire.

19. Revue fondée en 1978 par l'organisme Communication Jcunesse. Publiée depuis 1980 par l'Association Lurelu, société à but non lucratif.

20. «La critique à Lurelu est effectuée dans $95 \%$ des cas par des bibliothécaires professionnelles qui connaissent le livre pour la jeunesse et qui connaissent les enfants.» (Grégoire et Soulières 1986, p. 17) 


\subsubsection{Reconnaissance de la traduction dans le discours critique}

Nous avons pu dégager trois types de commentaires: ceux qui occultent la traduction; ceux qui sont axés sur la traduction; et, la via media, ceux qui prennent en compte le contenu et la traduction. Quelques exemples suffiront à illustrer les deux premières attitudes.

L'étape de la traduction est passée sous silence dans la recension des Chemins de la liberté dans In Review: «Ce roman se lit bien et il est bien écrit» (Maistrelli, 1979). Attitude tout à fait similaire que celle de la commentatrice de En montant à Low dans Lurelu: "Ce récit est à recommander autant pour la qualité de son écriture que pour l'originalité de son contenu.» (Dolbec 1986) Certains critiques font des remarques d'ordre stylistique sans établir de distinction entre l'original et la traduction. Une recension de Cher Bruce Springsteen de Kevin Major vante la "clarté d'écriture» et le "style grandement teinté d'humour et de réalisme» (Grégoire, 1991). Ce qui équivaut à donner un coup de chapeau à la traductrice, Marie-Andrée Clermont qui, tout le long de ce roman-journal intime, avait à "traiter» le sociolecte du narrateur, adolescent terre-neuvien ${ }^{21}$. Quant aux commentaires axés sur la traduction, ils s'annoncent dès le titre: "Une traduction centrée sur l'action» (Myers 1989), «Une traduction très adroite» (Myers 1991).

Notre dépouillement montre que les critiques qui comportent un discours sur la traduction sont quantitativement les moins nombreuses. Dans plus de la moitié des cas, le texte traduit est évalué comme un original. Parmi les critiques qui prennent en compte le statut de traduction du texte, l'attitude la

21. Voir la critique anglophone sur la langue de Kevin Major: «The first person narrative is enhanced by the delightful Newfoundland dialect that hastens a feeling of intimacy and involvement in the reader." (Mc Donough 1979). "He [Major] has an ear for speech patterns, and his language has not only the realistic saltiness of boisterous teenagers but also the regional flavour of Newfounland dialect.» (Engkent 1986) 
plus commune est de porter un jugement global sur le travail du traducteur. Ainsi pour Jasmine: «Gagnant du prix Ruth Schwartz en 1983, ce roman de Jan Truss a été traduit avec bonheur par Marie-Andrée Clermont.» (Prescott 1986). Ou pour Emily, titres pour lesquels Danielle Thaler, qui a choisi de centrer son analyse sur l'ambiguité du texte de L.M. Montgomery, ne manque pas de rendre hommage à la compétence de la traductrice: «Paule Daveluy qui avait traduit le premier volume s'est également attaquéc au second et a surmonté les obstacles avec une sûreté et une finesse qui ont depuis longtemps fait leurs preuves.» (Thaler 1991) Quant aux commentaires qui font de la traduction le principal objet du discours, ce sont les plus rares et on les trouve le plus souvent sous la plume d'universitaires.

\subsubsection{Paramètres d'évaluation}

Dans le corpus de critiques qui traitent explicitement les textes comme des traductions, nous avons reconnu un certain nombre de paramètres d'évaluation: fidélité; transparence; adéquation au public-cible; qualité linguistique; valeur littéraire. L'étude de l'importance relative de ces paramètres - dans les recensions individuelles et dans chacune des revues - fait apparaître chez les critiques de la littérature pour la jeunesse des «sourciers» et des "ciblistes" (Ladmiral 1986). La critique de Lurelu est de tendance cibliste, ne retenant souvent pour critère que la naturalisation, la «québécisation» de la traduction. La critique des deux revues canadiennes est majoritairement sourcière, préoccupée de la transmission fidèle de la lettre et de l'esprit du texte anglais. Quand elle s'interroge sur l'adéquation de la traduction au public cible, ce sont la tranche d'âge, le niveau scolaire et la langue maternelle du lecteur qui sont pris en compte, mais non son appartenance socio-culturelle.

Dans les rubriques qui suivent, nous illustrons chacun des paramètres d'évaluation par quelques exemples.

\section{Fidélité:}

- Jacob Deux-Deux et le vampire masqué: «La traduction suit de très près la version originale; elle se lit avec aisance, mais le piquant 
de l'aventure se reflète mieux dans la version anglaise.» (Aubrey 1979)

- La Passion de Blaine: «Le rythme de l'action a été capté avec délices dans la traduction" (Myers 1991).

- Deux grands ducs dans la famille et la Malédiction du tombeau viking: "Les traductions de Paule Daveluy et de Maryse Côté correspondent parfaitement au style des œuvres originales." (Hesse 1983)

- Le Programme Minerve: «la traduction ne respecte pas le sens profond de l'anglais [...] cela affecte la perception de la psychologie des personnages» (Myers 1989).

La pertinence des interventions du traducteur est jugée en fonction de ce paramètre:

- Deux grands ducs dans la famille: «La traduction substitue le présent pour (sic) le passé, ce qui renforce la vivacité des aventures racontées.» (Hesse 1983)

- Le Programme Minerve: «Toutefois nous avons trouvé le choix du présent en français regrettable. La version originale est écrite au passé et la lecture en est plus palpitante.» (Myers 1989)

\section{Transparence:}

- Premicr printemps sur le Grand Banc de Terre-Neuve: «La traduction de Maryse Côté est excellente et, comme telle, réussit sans peine à faire oublier au lecteur qu'il lit une traduction.» (Gaulin 1984)

- Jasmine: La traductrice «exécute l'exploit d'exprimer l'ambiance de l'original de façon si naturelle que n'importe quel lecteur non averti jurerait que Jasmine avait (sic) été rédigé en français.» (Rosmarin 1988)

Adéquation du ou des registres a) au registre de l'original; b) aux besoins du public-cible

Comme le maniement des registres constitue une difficulté particulière dans la traduction pour la jeunesse, nous lui consacrons une rubrique spéciale. Le choix du ou des registres est évalué tour à tour du point de vue de la source et de la cible. Il est clair que la sous-rubrique a) aurait pu être traitée sous la rubrique «fidélité». 
a. - Dans le Programme Minerve, le lexique est jugé plus soutenu que dans l'original: «ll s'agit donc de faire la mise en question du registre de langue choisi pour la traduction qui sans doute constituait un des défis de cette traduction. Le choix du mot «escogriffe» pour rendre «dumb", alors que crétin, bêta ou idiot aurait très bien fait l'affaire [...]» (Myers, 1989).

- Moi et Luc, à propos des registres exprimant la différence homme/femme: «Paule Daveluy a su traduire très exactement ces reflets purements linguistiques" (Paré, 1992).

- La Vie facile: «Le vocabulaire utilisé par Michelle Robinson facilite la lecture. Le niveau de langue des personnages caractérise bien leur statut social.» (Trudel 1992)

- La Main de Robin Squires: «Quelquefois le traducteur ne respecte pas le niveau de langue, ce qui s'exprime par des phrases bancales. L'on trouve, par exemple, un style très élégant dans une partie de la phrase et une forme très familière dans l'autre partie, avec, à la limite, un style non châtié.»

«Nous avons également des doutes, quant à l'équivalence des niveaux de langue de l'anglais au français dans les paroles des personnages.» (Myers 1986)

- Deux grands ducs dans la famille: «Le style est un peu trop soigné; l'emploi d'expressions familières l'aurait allégé: la langue de Farley Mowat est très vivante.» (Ledoux 1980)

b. - Le Programme Minerve: «La traductrice voulait peut-être simplifier le texte destiné à des jeunes de 11 à 14 ans, mais elle ne respecte pas les intentions de l'auteure.» (Myers 1989)

- Jours de terreur: «En ce qui concerne le niveau de langue, la traduction de Paule Daveluy est parfaite pour des jeunes francophones. [...] Pour les étudiants du programme d'immersion, le niveau semble un peu trop recherché [...].» (Lewin 1984).

- La Promesse de Luke Baldwin: «La traduction est bonne et rend bien le climat du roman. Les mots sont choisis, le vocabulaire riche, tout en étant à la portée des jeunes.» (Thériault-Houle 1980) 
Qualité de la langue-cible: choix lexicaux, anglicismes, impropriétés

La recension de Premier printemps apprécie le vocabulaire «riche, nuancé et correct», les «tournures élégantes et expressives» dont les lecteurs "pourront faire leur profit dans la vie courante" (Gaulin 1984). Un commentaire sur la traduction de la Passion de Blaine pose le problème de la traduction des régionalismes, toujours avec une visée pédagogique: «préférer le mot propre ou utiliser un terme permettant la compréhension par un public plus vaste» (Myers 1991).

Les anglicismes sont régulièrement signalés. Il est intéressant de noter que le discours critique tente de les expliquer ou de les excuser:

- La Passion de Blaine: «Si l'on note quelques anglicismes ici et là, ils ne semblent pas ternir l'ensemble, car ils sont du genre qui prévalent dans la langue parlée en français canadien dans certaines régions.» (Myers 1991)

- Maman va t'acheter un moqueur: «Un certain nombre d'anglicismes et de constructions étranges que les correcteurs d'épreuves auraient dû rectifier» (Paré 1988).

\section{Valeur littéraire de la traduction}

Ce paramètre d'évaluation globale est présent dans une partie du discours critique qui envisage le travail de traduction comme une variante de l'écriture pour la jeunesse. Le talent créateur des traductrices de Farley Mowat, Maryse Côté et Paule Daveluy, est souligné (Hesse 1983). Selon Marie J. Myers, la traductrice de la Passion de Blaine, Marie-Andrée Clermont, a réussi un «tour de force»: «certains passages sont rendus de façon si adroite qu'ils en deviennent d'une beauté incomparable» (Myers 1991). Il semble même que certains critiques attribuent à la traduction un effet mélioratif ${ }^{22}$. À propos du même roman, un critique conclut:

22. Sur cet effet mélioratif de la traduction, voir l'entrevue de Paule Daveluy, mentionnée plus haut: "On essaie aussi d'avoir des contacts avec ces auteurs qui lisent ou font lire leur œuvre en français. Il est déjà arrivé que l'un d'eux déclare: le texte français est meilleur que le texte anglais...» (Robin 1981, p. 16). 
«Le récit s'appuie sur une langue impeccable, fruit d'une traduction qui enrichit et avive les différents épisodes.» (Plaisance 1990) N'est-ce pas le même sentiment qui transparaît dans une recension d'Emily, lourde d'implicite: «À noter la traduction impeccable de Paule Daveluy, dont l'œuvre a été couronnée de nombreux prix et qui nous rend si attachant un personnage qui aurait pu être exécrable»? (Guindon, 1990)

\section{Adaptation à la culture-cible}

- Mike, chasseur de ténèbres: "Quant à la traduction très "québécoise" de Paule Daveluy, elle actualise le récit et le rend accessible aux jeunes d'ici.» (Vinet 1986) ${ }^{23}$

- Émilie 2 et 3: Le commentaire allusif, cité au point précédent, sur ce personnage "qui aurait pu être si exécrable" et que la traduction «nous rend si attachant» (Guindon 1990) ne laisserait-il pas entendre que la célèbre héroïne de L.M. Montgomery a besoin d'être naturalisée pour gagner le cour des jeunes lecteurs du Québec? Dans les deux cas, le roman de Hughes et celui de Montgomery, la traductrice se voit attribuer un rôle de médiatrice culturelle.

- Café Paradiso: «Si ces nouvelles avaient au moins été racontées dans une langue vivante, à travers des personnages crédibles, $j$ 'aurais pu m'y laisser transporter, mais le ton ancien et très américain m'a plutôt ennuyée.» (Guindon 1991)

La critique s'étonne alors du succès du livre en milieu anglophone, ct conclut: «Faut croire que le monde francophone se distingue là aussi du monde anglophone.»

L'attitude de Lurelu sur ce point n'est toutefois pas aussi monolithique que le laisseraient penser les commentaires précédents. La distance historique et géographique n'est pas perçue comme un obstacle dans la critique d'Une ombre dans la baie: «Les adolescents d'aujourd'hui s'identifieront à Mary, quinze

23. Le récit de Monica Hughes se situe dans l'Alberta des années 80. Il traite de la lutte d'un adolescent contre la leucémie, de ses amours et de ses amitiés, de son goût pour les sports et de sa passion pour la chasse. On peut se demander dans quelle mesure un tel récit a besoin d'être "actualisé» pour devenir accessible aux jeunes Québécois. 
ans, qui se débat avec ce questionnement, même si elle vient d'un autre pays: l'Écosse et d'une autre époque: 1822.» (Brodeur 1990) Quelques autres critiques prennent également soin de situer leur discours par rapport aux objectifs de la collection.

À ce stade de notre étude, le discours critique sur la collection «Les Deux Solitudes Jeunesse» présente donc les caractéristiques suivantes:

- Le statut de traduction du texte est, dans plus de la moitié des cas, passé sous silence.

- L'étude des aspects formels (linguistiques, stylistiques, rhétoriques) n'établit pas toujours de distinction claire entre le texte de départ et le texte d'arrivée. Les concepts les plus utilisés - ton, ćcriture, niveau de lecture, style même - peuvent, dans l'emploi qui en est fait, s'appliquer au texte anglais ou au texte français. Citons, en plus des exemples apportés plus haut, des syntagmes tels que: «clarté d'écriture», "style grandement teinté d'humour et de réalisme», "ton simple et accessible dans sa globalité».

- Dans les commentaires axés sur la traduction, le premier paramètre d'évaluation est la fidélité au texte anglais.

- Le discours critique perd rarement de vue la visée éducative de la collection. L'accessibilité du texte au public-cible est prise en compte. Mais la définition de cette accessibilité varie seulement les revues.

- Dans les critères d'accessibilité, la critique québécoise introduit des paramètres socio-culturels. La traduction doit comporter une «québécisation» du texte. Le récit traduit semble soumis aux mêmes paramètres d'évaluation que le récit écrit en français au Québec. Or le roman québécois pour la jeunesse des années 80 est très majoritairement le roman d'autoreprésentation de la jeunesse québécoise urbaine de la classe moyenne (Le Brun 1992). De là ce sentiment qu'un récit se déroulant à la même époque en Alberta doit être "actualisé»? La réflexion sera à poursuivre dans un cadre plus vaste, en prenant en compte les études sociocritiques sur la traduction québécoise (Simon 1989; Brisset 1990). 
Notre observation des textes nous a déjà permis de repérer divers degrés d' «acclimatation» des textes. Certains choix de traduction se trouvent ainsi en parfaite congruence avec le discours critique de Lurelu.

\section{Conclusion: La traduction entre la distanciation et l'assimilation}

Nous terminerons cette étude par quelques réflexions sur l'évolution de la conception de la traduction, du programme de la première directrice en 1980 aux choix de traductions illustrés par quelques titres des années 1990.

En comparant la décennie 80 et le début des années 90 du point de vue des objets discursifs, nous avons noté une évolution du discours de la différence - une autre histoire, d'autres lieux géographiques (l'Ouest, les Maritimes) suscitant un autre mode de vie - au discours de l'identité/communauté d'expérience de la jeunesse. Parallèlement apparait, dans quelques titres récents, un mode de traduction assimilateur. La différence est gommée tout d'abord dans ses manifestations les plus aisément repérables: la toponymie et l'anthroponymie. La traductrice du Fantôme de Val-Robert (1990) s'est efforcée d'effacer tout indice linguistique anglo-saxon. «Sullivan Town» devient "Val-Robert", petite ville peuplée de Gagnon et de Larocque. Dans Café Paradiso on observe un phénomène, nouveau dans la collection: les interventions pratiquées sur l'intertexte. L'épigraphe original, qui était une citation de «Chinese Café» de Joni Mitchell, devient "Je t'attends au café Rimbaud. Je sais que tu ne viendras pas» signé Lucien Francouur. Il sera intéressant d'observer la traduction des références culturelles dont est truffé le recueil et le traitement des différents degrés d'intertextualité. Sont gardés Vacances romaines avec Audrey Hepburn, "Petite fleur» de Sydney Bechet et «Michelle» des Beatles, mais non Joni Mitchell. Où finit la culture partagée? A quelles règles obéissent les équivalences culturelles?

La traduction de la littérature pour la jeunesse au Canada, d'une langue officielle à l'autre, soulève d'intéressantes questions d'interdiscursivité. En analysant cette collection à 
vocation didactique dans un contexte politique bilingue, de la sélection des textes aux principes de traduction, nous ne pouvons esquiver la classique question: traduit-on pour faire connaître et apprécier la différence ou pour la niveler? La traduction révèle-telle ou occulte-t-elle? Peut-être les enjeux de l'entreprise échappent-ils à ses promoteurs. Que parvient-il au juste, dans le discours global de la littérature pour la jeunesse québécoise, de ces "œuvres marquantes" d'auteurs canadiens-anglais? Les expériences de «naturalisation», d'«acclimatation» des romans anglais nous paraissent entrer en contradiction avec les premiers objectifs de la collection. Il nous paraît également significatif qu'une partie de la critique place la «québécisation» parmi les premiers paramètres d'évaluation de la traduction.

Parmi les aspects de l'étude qui nous paraissent mériter un approfondissement, l'étude de la traduction des références culturelles figure en bonne place. Beaucoup de romans traduits dans cette collection se réclament explicitement de la tradition littéraire britannique, celle de la littérature générale ou celle de la littérature pour la jeunesse $e^{24}$. Quelques titres parus dans les années 80 reposaient sur un intertexte qui a été fidèlement transmis en traduction. Jasmine de Jan Truss constitue un exemple caractéristique. Le point de départ de l'intrigue est en effet un poème de John Keats, placé en exergue, «Meg's Merrilies», dont Marie-Andrée Clermont donne une traduction fort adroite. La décision de traduire englobait alors l'acceptation de transmettre une certaine culture littéraire anglophone. Dans des parutions récentes, la traduction de la culture passe par la recherche d'équivalences dans la société-cible. L'étude du traitement de l'intertexte en traduction devrait mettre en lumière d'autres lignes de circulation - et quelques points de blocage - entre les «Deux Solitudes".

Université Concordia

24. Filiation revendiquée entre, par exemple, $A$ Handful of Time/ Du temps au bout des doigts de Kit Pearson et Tom's Midnight Garden de Philippa Pearce; Who is Frances Rain? / La Mystérieuse Frances Rain de Margaret Buffie et Alice's Adventures in Wonderland de Lewis Carroll. 


\section{Références}

AUBREY, Irene E. (1979). «Jacob Deux-Deux et le vampire masqué». In Review, vol. 13, $\mathrm{n}^{\circ} 1$ (février), p. 48.

ATKINSON, D.W. (1979). «History as Fiction». CCL, $\mathbf{n}^{\circ} 14$, pp. 6164.

23/24, pp. 28-39.

(1981). «Canadian Historical Fiction». CCL, $\mathrm{n}^{\circ}$

BLODGETT, E.D. (1982). Configuration: Essays in the Canadian Literatures. Downsview, ECW Press.

(1991). «Towards a Model of Literary Translation in Canada». TTR, vol. IV, $\mathrm{n}^{\circ}$ 2, pp. 189-206.

BRISSET, Annie (1990). Sociocritique de la traduction: Thêâtre et altérité au Québec. Longueuil (Québec), Le Préambule.

BRODEUR, Micheline (1990). «Une ombre dans la baie». Lurelu, vol 12, n 3 (hiver), pp. 11-12.

CCL. Canadian Children's Literature/Littérature canadienne pour la jeunesse. University of Guelph, Departments of English and French, Guelph, Ontario.

CHARETTE, Christiane (1985). «Des traductions». Lurelu, vol. 8, $\mathrm{n}^{\circ} 1$, pp. 30-31.

CLERMONT, Marie-Andrée (1980). Alerte au lac des loups. Montréal, Fides, collection du Goéland.

(1984). Visiteurs extra-terrestres. Saint-

Lambert, Héritage, collection Galaxic. Traduction de Beckoning Lights (1982) de Monica Hughes. 
DAVELUY, Paule (1959). Un été enchanté. Montréal, Éditions de l'Atelier. Traduction par Monroe Stearns (1962): Summer in VilleMarie. New-York, Holt, Rinehart and Winston.

(1980 a). «Une richesse nouvelle: la collection des Deux Solitudes/Jeunesse». CCL, $\mathbf{n}^{0}$ 18-19, pp. 5-9.

(1980 b). «Les Livres canadiens pour la jeunesse en traduction». In Review, vol. 14, n 4 (août), pp. 14-17.

(1985). «Un autre ami nous quitte: Claude Aubry». CCL, no 38 , pp. 66-67.

DENTON, Vivienne (1983). «History and Myth». CCL, $\mathrm{n}^{\circ} 29, \mathrm{pp}$. 63-65.

DOLBEC, Denise (1986). "En montant à Low». Lurelu, vol. 9. $\mathrm{n}^{\circ}$ 3 (hiver), p. 15.

EGOFF, Sheila et Judith SALTMAN (1990). The New Republic of Childhood. A Critical Guide to Canadian Children's Literature in English. Toronto, Oxford University Press.

ENGKENT, Gary (1986). «Kevin Major: A Writer to Watch». CCL, $n^{\circ} 43$, pp. $56-57$.

GAGNON, André (1987). «Translations of Children's Books in Canada». $C C L, \mathrm{n}^{\circ} 45, \mathrm{pp}$. 14-53.

GAULIN, Michel (1984). "Aventure et responsabilité». $C C L, \mathrm{n}^{\circ}$ 35/36, pp. 126-128.

GRÉGOIRE, Josée (1991). "Cher Bruce Springsteen». Lurelu. vol. $13, \mathrm{n}^{\circ} 3$ (hiver), p. 21.

GRÉGOIRE, Madeleine et SOULIĖRES, Robert (1986). «Tribune libre: (Ne) tirez (pas) sur le critique», Lurelu, vol. 6, $\mathrm{n}^{\circ} 3$ (hiver), pp. $17-21 ; 32$. 
GUINDON, Ginette (1990). «Émilic, tomes 2 et 3». Lurelu, vol. 13, $\mathrm{n}^{\circ} 2$ (automne), p. 10.

(été), p. 21.

(1991). «Café Paradiso». Lurelu. vol. 14, n 1

HESSE, M.G. (1983). «Farley Mowat, ami des jeunes». $C C L, \mathrm{n}^{\circ}$ 31/32, pp. 123-126.

HUARD, Michèle (1982). «Les Éditions Pierre Tisseyre». Lurelu, vol. $5, n^{\circ} 3$ (automne), pp. 22-23.

In Review. Canadian Books for Young People. Libraries and Community Information Branch, Toronto.

LADMIRAL, Jean-René (1986). «Sourciers et ciblistes», Reoue d'esthétique, $\mathrm{n}^{\circ} 12$, nouvelle série, pp. 33-42.

LE BRUN, Claire (1992). «Mais où sont passés les pères? Un cas de censure sociale dans la littérature québécoise pour la jeunesse des années 80». CCL, n 68, pp. 99-113.

(1993). «Edgar Alain Campeau et les autres: le lecteur fictif dans la littérature canadienne pour la jeunesse (19861991)». Voix el Images, vol. XIX, n 1 (automne), pp. 151-165.

LEDOUX, Danielle (1980). «Deux grands ducs dans la famille». In Review, vol. 14, $\mathrm{n}^{\circ} 6$ (décembre), pp. 50-51.

LEMIEUX, Louise (1972). Pleins feux sur la littérature de jeunesse au Canada français. Montréal, Leméac.

LEPAGE, Françoise (1980). «La Malédiction du tombeau viking». In Review, vol. 14, $\mathrm{n}^{\circ} 5$ (octobre), p. 56.

LEWIN, Louise (1984). «Un roman historique». $C C L, n^{\circ} 35 / 36$, pp. 140-143. 
Lurelu. La seule reoue exclusivement consacrée à la littérature québécoise pour la jeunesse. Association Lurelu, Montréal.

MAISTRELLI, Gloria (1979). «Les Chemins secrets de la liberté». In Review, vol. 13, $\mathrm{n}^{\circ} 5$ (octobre), p. 56.

MARTIN, Ruth V. (1992). Norms of the Translated Novels: Canada 1967-1982. University of Alberta, Ph. D. Dissertation (non publiée).

(A paraître). "Genre types and political discourse in the translated Canadian literatures: 1972-1992». Communication présentée au colloque de l'Association canadienne de traductologie, Ottawa, Université Carleton, juin 1993.

MCDONOUGH, Irma (1979). «Hold Fast». In Review, vol. 13, $n^{\circ}$ 5, pp. 70-71.

MCLENNAN, Hugh (1945). Two Solitudes. Toronto, Collins.

MEZEI, Kathy (1988). Bibliography of Criticism on English and French Literary Translations in Canada (1950-1986 Annotated)/Bibliographie de la critique des traductions littéraires anglaises et françaises au Canada (de 1950 à 1986 - avec commentaires). Ottawa, Les Presses de l'Université d'Ottawa/Fédération canadienne des études humaines, 1988.

MYERS, Marie J. (1986). «Le Mystère de l'île au chêne». CCL, $\mathbf{n}^{\circ}$ 44, pp. 54-56.

$\overline{54, \text { pp. 79-80. }}$

(1989). «Une traduction centrée sur l'action». CCL, $\mathrm{n}^{\circ}$ (1991). «Une traduction très adroite». $C C L, \mathrm{n}^{\circ} 61, \mathrm{pp}$.

$\overline{84-85 .}$

PAQUETTE, Réal (1982). «La Traduction». Lurelu, vol. 5, $\mathrm{n}^{\circ} 1$ (printemps), pp. 3-6. 
PARÉ, François (1988). «Mini-comptes rendus». $C C L, \mathrm{n}^{\circ} 51, \mathrm{p}$. 103.

109-110.

(1992).«Mini-comptes rendus». CCL, $\mathrm{n}^{\circ} 67, \mathrm{pp}$.

PLAISANCE, Gilbert. «La Passion de Blaine». Lurelu, vol. 12, $\mathrm{n}^{\circ}$ 3 (hiver), p. 9.

POULIN, Monique (1987). «Maric-Andréc Clermont, auteure». Lurelu, vol. 9, no 3 (hiver), pp. 20-21.

PRESCOTT, Monique (1986). “Jasmine». Lurelu, vol. 9, $\mathrm{n}^{\circ} 3$ (hiver), pp. 14-15.

ROBIN, Marie-Jeanne (1981). «Rencontre avec Paule Daveluy». Lurelu, vol. 4, n 1/2 (printemps-été), pp. 16-17.

ROSMARIN, Léonard (1988). «Le Triomphe de l'échec». CCL, $\mathrm{n}^{\circ}$ 51, pp. 95-97.

THALER, Danielle (1991). «La Conquête de l'écriture», $C C L, \mathrm{n}^{\circ}$ 61, pp. 72-74.

SHEK, Ben-Z. (1988). «Diglossia and Ideology: Socio-cultural Aspects of "Translation" in Quebec". TTR, vol. 1, $\mathrm{n}^{\circ} 1$, pp. 85-91. (1991). French-Canadian \& Québécois Novels. Toronto, Oxford University Press.

SIMON, Sherry (1989). L'Inscription sociale de la traduction au Québec. Québec, OLF/Gouvernement du Québec.

STRATFORD, Philip (1977). Bibliography of Canadian Books in Translation: French to English and English to French/Bibliographie de livres canadiens traduits de l'anglais au français et du français à l'anglais. Ottawa, HRCC/CCRH. 
(1986). All the Polarities: Comparative Studies in Contemporary Cnadian. Novels in French and in English. Toronto, ECW.

THÉRIAULT-HOULE, Mariette (1980). «La Promesse de Luke Baldwin". In Review, vol 14, nº 6 (décembre), pp. 36-37.

TRUDEL, Marc-Alexandre (1992). «La Vie facile». Lurelu, vol. 15, $\mathrm{n}^{\circ} 2$ (été), pp. 13-14.

VINET, Isabelle (1986). «Mike, chasseur de ténèbres». Lurelu, vol. $9, \mathrm{n}^{\circ} 2$ (automne), p. 25.

\section{ANNEXE}

Les romans canadiens-anglais pour la jeunesse en traduction québécoise dans la collection des "Deux Solitudes/Jeunesse" (Montréal, Éditions Pierre Tisseyre):

ALLEN, R. The Violin 1976

Le Violon Claire Martin 1976

ATWOOD, M. Ulp in a Tree 1978

Sur un arbre perchés Michel Caillol 1979

BELL, W. Forbidden City 1990

Shan da et la cité interdite Paule Daveluy 1991

BROOKS, M. Paradise Café and Other Stories 1988

Café Paradiso et autres nouvelles Marie-Andrée Clermont 1990

BUFFIE, M. Who is Frances Rain? 1987

La mystérieuse Frances Rain Martine Gagnon 1991

CALLAGHAN, M. Luke Baldwin's Vow 1948

La promesse de Luke Baldwin Michelle Tisseyre 1980

CLARK, J. The Hand of Robin Squires 1977

La Main de Robin Squires Claude Aubry 1984 
CRADDOCK, S. The TV War and Me 1980

Les Commandos de la télé Cécile Gagnon 1991

DOYLE, B. Easy Avenue 1988

La Vie facile Michelle Robinson 1991

DOYLE, B. Up to Low 1982

En montant à Low Claude et Danielle Aubry 1986

DOYLE, B. You can pick me up at Peggy's Cove 1979

Je t'attends à Peggy's Cove Claude Aubry 1982

FREEMAN, B. First Spring on the Grand Banks 1978

Premier printemps sur le Grand Banc de Terre-Neuve Maryse Côté 1983

FREEMAN, B. The Last Voyage of the Scotian 1976

Le dernier Voyage du Scotian Maryse Côté 1982

GERMAN, T. A Breed Apart 1985

Une race à part Maryse Côté 1988

HALVORSON, M. Dare 1980

Comme un cheval sauvage Paule Daveluy 1992

HUGHES, M. Blaine's Way 1986

La Passion de Blaine Marie-Andrée Clermont 1989

HUGHES, M. Hunter in the Dark 1982

Mike, chasseur de ténèbres Paule Daveluy 1985

KROPP, P. The Rock 1989

Solide comme roc Marie-Andrée Clermont 1991

LITTLE, J. Listen for the Singing 1977

Écoute, l'oiseau chantera Paule Daveluy 1980

LITTLE, J. Mama's going to buy you a mockingbird 1984 
Maman va t'acheter un moqueur Paule Daveluy 1986

LUNN, J. Shadow in Hawthorn Bay 1986

Une ombre dans la baie Paule Daveluy 1989

MACKAY, C. The Minerva Programme 1984

Le Programme Minerve Marie-Andrée Clermont 1987

MACKAY, C. Mini-bike Rescue 1982

La Fille à la mini-moto Michelle Tisseyre 1984

MAJOR, K. Dear Bruce Springsteen 1987

Cher Bruce Springsteen Marie-Andrée Clermont 1989

MAJOR, K. Far from Shore 1980

Loin du rivage Michelle Tisseyre 1989

MAJOR, K. Hold Fast 1978

Tiens bon! Michelle Robinson 1984

MONTGOMERY, L. M. Emily of the New Moon 1925

Émilie de la Nouvelle Lune Paule Daveluy 1983-1988

MOWAT, F. Owls in the Family 1961

Deux grands ducs dans la famille Paule Daveluy 1980

MOWAT, F. The Black Joke 1962

Une goélette nommée Black Joke Michel Caillol 1982

MOWAT, F. The Curse of the Viking Grave 1966

La Malédiction du tombeau Viking Maryse Côté 1980

O'HEARN, A. Me and Luke 1987

Moi et LuC Paule Daveluy 1990

PEARSON, K. A Handful of Time 1987

$D u$ temps au bout des doigts Hélène Filion 1990

PEARSON, K. The Sky Is Falling 1989 
RICHLER, M. Jacob Two-Two Meets the Hooded Fang 1975

Jacob Dcux-Deux et le vampire masqué Jean Simard 1977

SMUCKER, B. Amish Adventure 1983

Un monde hors du temps Paule Daveluy 1985

SMUCKER, B. Underground to Canada 1977

Les Chemins secrets de la liberté Paule Daveluy 1978

SMUCKER, B. Days of Terror 1979

Jours de terreur Paule Daveluy 1981

SPENCER, B. The Ghost of Sullivan Town 1984

Le Fantôme de Val-Robert Martine Spencer 1990

TRUSS, J. Jaswin 1982

Jasmine Marie-Andrée Clermont 1986

WIELER, D. Bad Boy 1990

Le Bagarreur Marie-Andrée Clermont 1991

WILSON, E. Summer of Discovery 1984

Terreur au bivouac Michelle Tisseyre 1987

RÉSUMÉ: La Littérature canadienne-anglaise pour la jeunesse en traduction québécoise: analyse discursive de la politique éditoriale et de la réception critique des «Deux Solitudes/Jeunesse» (1980-1992). La collection «Les Deux Solitudes/Jeunesse", publiée aux éditions Pierre Tisseyre à Montréal, est un cas exemplaire de traduction de littérature canadienne-anglaise pour la jeunesse en français, puisque c'est la seule qui se donne pour objectif de faire connaître au Québec les "œuvres marquantes» du Canada anglais. L'étude fait l'historique de la collection (1980-1992) et analyse le discours justificatif et les choix éditoriaux. Le contenu discursif des textes traduits est comparé à celui des textes publiés en français au Québec durant la même période. La dernière partie examine la réception critique 
de la collection dans les revues spécialisées: la reconnaissance ou non du statut de traduction du texte, les paramètres d'évaluation utilisés par les critiques. La conclusion met l'accent sur une évolution, dans les choix de traduction comme dans la réception critique, du discours de la différence vers un discours de la communauté d'expérience des jeunes. Cette tendance assimilatrice est particulièrement perceptible dans la traduction des renvois intertextuels et des références culturelles, dont l'analyse est esquissée et qui sera poursuivie dans un autre cadre.

ABSTRACT: English-Canadian Children's Literature translated in Québec: Discourse Analysis of the Editorial Policy and Critical Reception of the Series "Deux Solitudes/Jeunesse" (1980-1992) - "Deux Solitudes/Jeunesse," published by Pierre Tisseyre in Montréal, provides a particularly interesting casestudy for the translation of English-Canadian children's literature into French, because it is the only series which has as its mandate to provide Québec with the most important works of English Canada. The article discusses the history of the series (1980-1992) and analyses the justifications for editorial choices. The content of the translated texts is compared to that of texts published in French in Québec during the same period. The last part examines the critical reception of the collection in specialized journals, in particular whether the text is recognized as a translation or not, and how evaluation criteria are used by the critics. The conclusion stresses the movement towards discourse - both in the choices of texts to translate and in critical reception - which underlines the common experience of young people, rather than the differences. The assimilative tendency is particularly noticeable in the translation of cultural and intertextual references. 